$a$

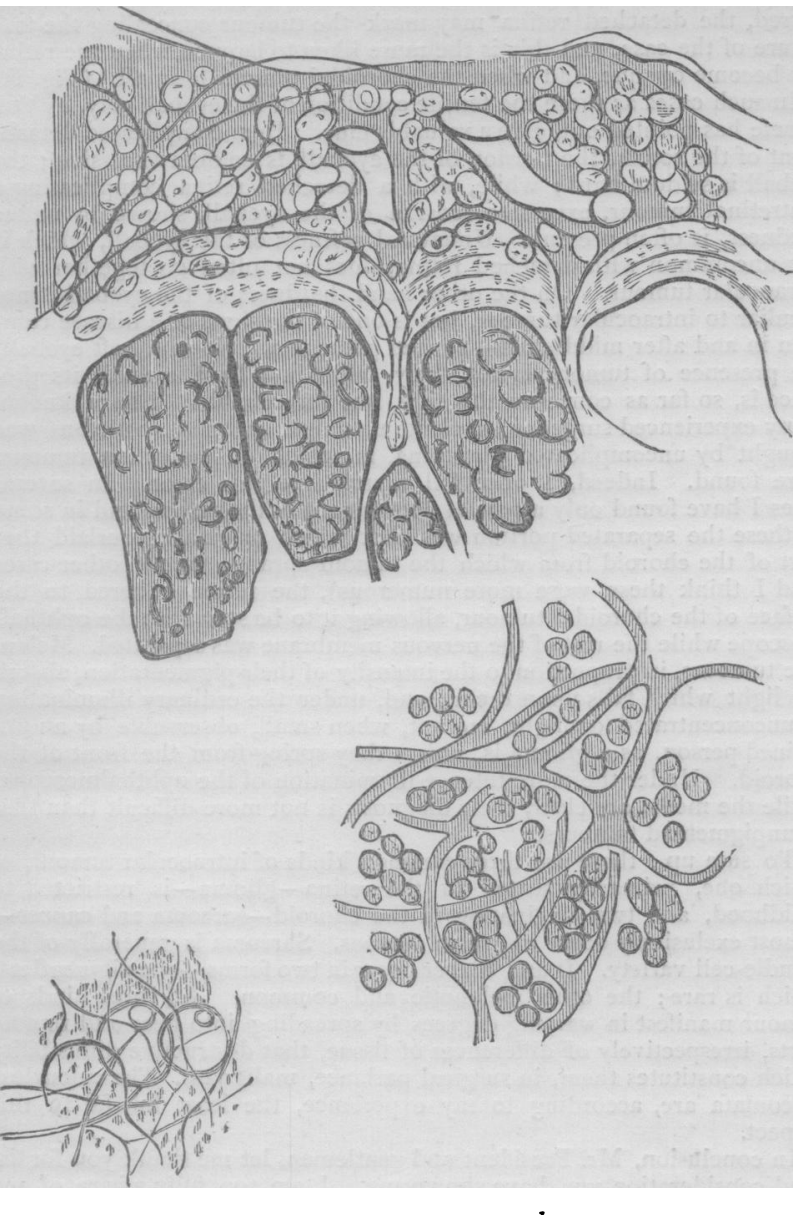

$c$

Fig. 4.- $a$. Cross section of an Inflamed Optic Nerve. The intestinal connective tissue between the inner and the outer sheaths is infiltrated with exudationcorpuscles. $b$. Portion of the interstitial tissue with exudation-cells exudation.

\section{ON STRANGULATION OF THE UTERUS.*}

By GRAILY HEWITT, M.D., F.R.C.P.,

Professor of Midwifery in University College; Physician-Accoucheur at University College Hospital; etc.

UNDER the term "strangulation" of the uterus, I propose to describe a pathological condition of that organ of great importance, hitherto, I believe, I will not say absoluteiy unrecognised, but unexplained as regards the mechanism of its occurrence, and certainly undervalued in regard to the influence which it exercises in the production of uterine suffering and the origination of some of the most troublesome maladies to which women are subject.

It is well known that, if one of the fingers be forcibly kept in a state of extreme flexion for a few hours, the result is, that the finger becomes swollen, congested, and painful ; the pressure due to this flexion impeding the circulation and producing these effects. Similarly, a bandage tied round the arm produces congestion and pain in the hand, even when the pressure is kept up for a short time only.

Now, the forcible bending of the uterus is attended with results of an analogous character. Flexion of this organ at its centre similarly interferes with the circulation in its tissues, leading to the effects now to be described under the term "strangulation" of the uterus.

* Read in the Midwifery Section at the Annual Meeting of the British Medical Association in Newcastle-upon-Tyne, August 1870.
A moment's reflection on the anatomy of the uterus and the arrangement of its vascular supply leads necessarily to the inference that any compressing force applied at or about the middle of the uterus will inevitably lead to obstruction in the circulation of the organ above that point; the result being an engorged congested condition of the body and fundus of the uterus. In a lesser degree, also, such compression will give rise to like results in the cervix and neighbourhood of the os uteri, where the circulation must be also materially interfered with. A reference to the beautifully executed illustrations of the vessels of the uterus in Dr. Savage's work On the Female Pelvic Organs (Plate VII, and ed.) will lend force to this explanation. Let it be supposed, for instance, that a ligature were applied, not very tightly, round the uterus at about its middle : the congestion referred to would occur just as certainly as when a bandage is applied round the arm in the operation of venesection.

Now, acute flexion of the uterus produces a result very analogous to that which would occur were a ligature applied round the uterus. If the organ be bent backwards, for instance, a constriction will occur at the seat of the flexion, which will be intense and severe in proportion to the degree of the flexion. The passage of blood to and from the fundus uteri is impeded from the simple fact that the opposite sides of the blood-vessels are brought into contact, and the passage of the blood obstructed. The bending of a tube composed of non-rigid materials necessarily produces more or less occlusion of that tube; and so it is with the vessels of the uterus when that organ is in a state of acute flexion.

Strangulation of the uterus is most intense when the organ is sharply bent backwards and assumes a shape like that of a chemical retort; and the strangulating effects are most marked when such flexion has been rather suddenly brought about. But it exists also, though to a less degree, when the uterus is sharply bent forwards; the reason for the difference residing in the circumstance that the anatomical relations of the uterus allow a more extensive and a sharper degree of flexion backwards.

I maintain, then, that a necessary result of acute flexion of the uterus is a strangulation of the organ, which will be characterised by various degrees of congestion, according to the rapidity with which the lesion is brought about, and which will be influenced by other circumstances too obvious to require special mention.

Congestion of the uterus due to strangulation, and brought about by the mechanism above described, will, and frequently does, aggravate and intensify the flexion, the two mutually increasing as the disease advances. The original cause of the flexion is various; but I do not hesitate to say that, in the numerous cases which have fallen under my notice, a mechanical pressure upon the fundus uteri from above was in almost-all cases demonstrable by careful scrutiny of the history of the individual case. An accidental increase in the size of the uterus, such as is present in weakly women for some weeks after parturition, or such as is present during menstruation, or from the presence of chronic congestion of the uterus from any other cause, - under any one of these conditions, an accidental slip, or fall, or strain, or unusual exertion of any kind, will supply the determining and actual cause of the flexion. Increased size of the uterus, then, coupled with a lax, pliable condition of the organ, is, from my point of view, the predisposition; while mechanical pressure from muscular exertion of the walls of the abdomen or other mechanical agent is the exciting cause of the flexion. The flexion, once produced, determines the effects which I describe under the term "strangulation".

Strangulation of the uterus is temporary or permanent, according to circumstances. The more ordinary circumstance is, that the uterus so affected is sometimes in what may be termed a condition of acute strangulation, which lasts for a certain time, but, on the patient taking rest and remaining quiet, the acute state passes off, leaving a less severe degree of congestion present. Undoubtedly, after the lapse of time, the flexed uterus becomes rather less liable to attacks of acute strangulation, if I may so express myself; but a sudden exertion of any kind may, even in cases of long standing, give rise to this degree of the affection.

An account of the symptoms of strangulation of the uterus will at the same time furnish an opportunity for explaining the relation between this condition and inflammation of the uterus.

In acute strangulation of the uterus, the patient complains of an in toierable pain in the back, which absolutely prevents motion. There may be tenderness on pressure over the hypogastric region, which is sometimes very sensitive indeed to the touch. On touching the uterus from the vagina, it is found extraordinarily sensitive; but this sensitiveness is found to be really almost limited to the displaced fundus, which can be felt behind the vagina, swollen, large, acutely sensitive to the slightest touch. The very acute cases are always cases of retroflexion. 
Occasionally pains coming and going, like small labour-pains, are observed.

And now I come to certain symptoms which are exceedingly interesting and important in their whole bearing, and which are very frequently present in cases of acute strangulation of the uterus : I mean the nervous and hysterical symptoms.

Clinical observation has led me to the conclusion that, in cases of what may be termed acute hysteria attended with violent paroxysma outhreaks, the pathological condition which lies at the root of the evil is strangulation of the uterus such as that above described. The truth of this theory, which, so far as I am aware, is a novel one as propounded in this particular way, will, I am convinced, become evident to those who take the pains to investigate and dispassionately inquire into the facts of individual cases. So much attention has been directed to the condition of the os and cervix uteri, to the exclusion, in too many instances, of the condition of the body and fundus of the uterus, that the connexion between these hysterical phenomena and marked flexion of the uterus leading, as I have explained, to strangulation of the organ, has been overlooked. I maintain that the connexion exists, and can be indubitably proved.

The nervous symptoms produced by acute strangulation of the uterus might be accurately described after an a priori method. Acute congestion of the body of the uterus, such as is produced by compression of the organ at its centre, must necessarily be accompanied by exaltation of the nervous susceptibilities of the uterus. The trunks of the nerves undergo, together with the blood-vessels, considerable pressure in flexion of the uterus; the result being an acutely sensitive condition of the body of the uterus. But the uterus is not simply unduly sensitive to the touch under such circumstances; its sensitiveness is felt in other organs of the body. Reflex movements and agitations of other parts of the body are excited by this irritation : hence the violent paroxysms seen in cases of acute hysteria. Let the uterus under the pressure of such symptoms be examined by the touch, it will be found extraordinarily sensitive to pressure. This sensitiveness is due to strangulationto a mechanical pressure, leading, as before explained, to interference with the normal circulation and innervation of the uterus.

No term so well as "strangulation" conveys an idea of the actual condition of the uterus in a patient at once the subject of acute flexion of the organ and liable to occasional severe hysterical attacks.

An acutely painful condition of the uterus does not invariably lead to hysterical attacks, though the converse proposition is undoubtedly true. In other words, the reflex phenomena are not always excited. But, in a chronic form, milder hysterical symptoms are very commonly present in cases where the flexion or displacement is less severe. In other words, hysterical symptoms in a milder form are pretty frequent when the uterus is slightly flexed or so displaced that the circulation is dis. arranged and partial strangulation occurs.

Anteflexion of the uterus is, according to my experience, a common condition in cases of chronic hysteria; the more severe cases of hysteria being associated with retroflexion of the uterus.

If I am asked for the proof of the truth of the theory now enunciated as to the connexion between these hysterical paroxysms and phenomena of various kinds and strangulation of the uterus, my reply is that, having attentively considered the matter, and having had the idea before my mind for the last three years, I can affirm that I have not yet met with any fact or series of facts which are not in strict consonance with the view now expressed. Did the time permit, cases abundantly illustrative are ready to be cited.

Regarding the connexion between strangulation of the uterus and inflammation of this organ - a part of the subject which I can only briefly allude to-the view which I have taken of the matter involves the conclusion that strangulation, or, as it might be termed, acute mechanical congestion, is the principal element in many cases of inflammation of the uterus; but, on the other hand, it is not to be denied that an inflamed, enlarged, softened condition of the uterus is not seldom the forerunner of strangulation. Further than this also, and to carry the case on to a later period of its history, this strangulation intensifies and increases the strangulation.

Regarding the treatment of strangulation of the uterus, and the important effects and complications often observed, there is necessarily much to be said ; but I must limit myself to an indication of the principles which have guided me, and have appeared to be reliable in the management of such cases.

As a general rule - a rule to which I have observed exceedingly few exceptions - this maintenance of the uterus in a proper shape by mechanical means-the restoration, in fact, of the organ to its normal relations, both in regard to the parts of the organ itself and the organs in the immediate vicinity, is attended with an abolition of the symptoms, or, at all events, with their immediate amelioration. In most cases, the relief is most instantaneous and striking. I could cite case after case of this kind. The hysterical cases are as striking as any in regard to the efficacy of the treatment.

To restore the uterus to its proper shape, and to maintain it in this condition, various mechanical devices, the sound, the intrauterine stem, the Hodge pessary, the "cradle" pessary, which latter is my invention, all prove useful in their appropriate places. It is undoubtedly a question, how far depletion is necessary in these cases of strangulation of the uterus. The good effects of unloading the vessels of a congested loaded uterus must be obvious enough, and leeches would occasionally be useful. But, on the supposition of the accuracy of the explanation above given of the pathological condition present, it must be plain that the first and foremost indication will be to straighten the uterus-to remove that flexion which is all the time operating in such a way as to close the vessels and give rise to continued severe congestion. Local depletion may be beneficial, but leaves, for the most part, the radical evil untouched.

\section{THE SUCCESS OF THE ANTISEPTIC TREATMENT OF WOUNDS EXPLAINED WITHOUT REFERENCE TO GERMS.}

\section{By R. T. MANSON, L.R.C.P.Ed., etc., Witton-le-Wear, Durham.}

HAVING adopted and found good reasons for continuing to use what is known as Professor Lister's treatment of wounds, I may go on to state that I entertain serious doubts as to the soundness of the theory upon which it is based. These doubts arose from some cases of wounds in which the "germs" had full access for some time, in which no germicide was used to the surface of the wound, and yet in which no pus was formed.

It is difficult to resist the conviction that the ubiquitous impalpable germ, as difficult to capture as the fairies of old, has, like the "good folks", been debited with much mischief of which it is innocent. Professor Lister's theory is so well known that it is unnecessary to recapitulate it. The theory which I propose is, that the success of Professor Lister's treatment is due to the carbolic acid producing on the surface of a wound a stratum of coagulum which protects the germinal cells below from the deleterious action of the oxygen of the atmosphere, just as the epidermis protects the true skin; and, secondly, to the production of an atmosphere or retention of an atmosphere of carbonic acid in and round the wound.

Whether pus-corpuscles be the descendants of nuclear cells or parasites within them, it is known that pus results from an abnormal growth of germinal or nuclear cell-material-that some obstacle to abnormal growth has been removed, and an increased access of pabulum allowed. In the case of a wound it is clear that the barrier, the scale armour of epithelium, is destroyed, and an increased supply of oxygen ad. mitted. The result is the formation of pus. If this be so, it is evident that if we restore, to as great an extent as we can, the normal condition, we shall be doing the best we can as surgeons. This, I take it, we do by applying the carbolic acid lotion to the surface of a wound. Put a drop of albumen, say white of egg, on a glass slide, and beside it a drop of carbolic acid lotion; observe them under a microscope; let the two drops touch, and at the point and instant of junction a thin film, of what we may call "formed material", coats the albumen, and if the two drops are mixed a solid white mass of coagulated albumen forms on the slide ; or, put a drop of saliva, containing epithelial cells, on the glass, and add solution of carbolic acid, the outline of the epithelial scale grows clearer, indicating the change produced. The carbolic lotion, then, changes the nuclear matter of each cell on the surface of the wound to which it is applied into an inert "formed material" protective of the vitalities below, as the epithelium does to what it covers. I need only point to the well-known fact that the degree of pus formation, and consequent pitting in small-pox, is in direct proportion to the exposure of the part to the action of the oxygen of the atmosphere, to show that such free access does act injuriously.

The primitive surgery of our boyhood, when properties of great virtue were believed to exist in saliva, and when licking or spitting upon a slight excoriation or cut was understood to be a remedy of undoubted efficacy, may have been founded in school-boy instinct; but its value would be explicable on the same principle.

On the value of an atmosphere of carbonic acid to a wound (and such atmosphere must of necessity be induced by the exclusion of common air), I say no more than to point out that it is the normal atmo. sphere of all covered tissues, and that the old plan of healing wounds by scabbing is the ancestor of Professor Lister's treatment. 